\title{
Coupling Power System Dynamics and Building Dynamics to Enable Building-to-Grid Integration
}

\author{
Yangyang $\mathrm{Fu}^{1} \quad$ Sen Huang ${ }^{2, *} \quad$ Draguna Vrabie $^{2} \quad$ Wangda Zuo $^{1}$ \\ ${ }^{1}$ Department of Civil, Environmental and Architectural Engineering, University of Colorado Boulder, Boulder, CO, \\ USA y yangyang.fu, wangda.zuo\} @colorado.edu \\ ${ }^{2}$ Pacific Northwest National Laboratory, Richland, WA, USA \{sen.huang, draguna.vrabie\}@pnnl.gov
}

\begin{abstract}
The interactions between power system dynamics and building dynamics are usually ignored or over-simplified in existing power system and building modeling and simulation tools, which limits how system modeling can support Building-to-Grid integration. This paper discusses a new approach to consider those interactions by modeling motor-driven building devices or systems. The motordriven model is based on simplified mechanical rotation equations and allows us to study the coupling relationship between frequency/voltage in the power system and motor-driven device operation. This model is validated by performing one proof-of-concept case study with Modelica. The simulation results suggest that the proposed model can yield better representations of these interactions than the existing simplified models, especially the ones with the fast transient dynamics.
\end{abstract}

Keywords: Building-to-Grid Integration, Motor, Coupling Simulation

\section{Introduction}

There are significant interactions between power system and the buildings. For example, research suggests that the supply voltage of the power system dramatically affects building energy efficiency (Hood, 2004; Bichik et al., 2015; Lee, 2014). On the other hand, building systems, especially heating, ventilation, and air conditioning (HVAC) systems, attribute to the voltage stability issues in the power system (Wu et al., 2006; He et al., 2012; Li et al., 2017). It is generally necessary to consider those interactions, when designing or operating power systems or buildings, to avoid undesirable side effects.

However, when simulating the power systems and buildings, those interactions are usually ignored. For instance, some power system models assume the power factors of the building system to be fixed (Chassin et al., 2008). In addition, most building modeling tools implicitly ignore the influence of power systems on buildings by assuming the supply voltage to be constant (Crawley et al., 2001). Some models do consider those interactions, but in a simplified manner. One example is the ZIP coefficient model (Bokhari et al., 2014), which represents the variation (with voltage) of a load as a composition of the three types of constant loads: impedance "Z", current "I", and power "P" loads. It calculates the active/reactive power of a device under varying voltage conditions, by

$$
\begin{aligned}
& P=P_{o}\left(Z_{p}\left(\frac{V}{V_{o}}\right)^{2}+I_{p} \frac{V}{V_{o}}+P_{p}\right) \\
& Q=Q_{o}\left(Z_{q}\left(\frac{V}{V_{o}}\right)^{2}+I_{q} \frac{V}{V_{o}}+P_{q}\right)
\end{aligned}
$$

where $P, Q$, and $V$ are the active power, reactive power, and voltage, respectively. Subscript $o$ denotes the rated condition while $p$ and $q$ denote the active and reactive power, respectively. Essentially, the ZIP coefficient model approximates the influence of the voltage on the device with a polynomial function.

It is noted that the above assumptions or simplifications regarding the interaction between power systems and buildings may be justified for certain applications. For example, for buildings where the resistive devices (such as the electric heater) dominate, the assumption that the power factor is constant may be valid. In addition, when considering a regulated power system, it might be acceptable to assume that the supply voltage is constant in evaluating building design or operation (Gilbert, 1965). Furthermore, the ZIP coefficient model was widely used in the static or semi-dynamic analysis on the power system (Hatipoglu et al., 2012).

Nevertheless, it is our view that the aforementioned assumptions or simplifications may be inappropriate for some applications, especially the Building-to-Grid integration activities. For instance, (Arriffin et al., 2017) proposed an approach to increase the energy efficiency of the buildings by optimizing the supply voltage. When evaluating this approach, it is necessary to consider how the buildings respond to the varying voltage in terms of active/reactive power and the quality of the service they provided to the occupants. It is also necessary to consider how the proposed approach affects the stability of the power supply. Apparently, none of the aforementioned assumptions or simplifications help users to do so.

In this paper, we provide a more realistic representation of the interactions between power grid and buildings by presenting a new motor-driven model. The model is based on simplified mechanical rotation equations. It allows us to consider not only the effect of thermal dynamics in the 
building side on motor operations, but also the influence from the power supply side on motor behaviors. Thus, it is more suitable for the Building-to-Grid integration activities as we discussed above.

The rest of this paper is organized as follows: we first describe the motor system and simplify it for modeling purpose; then we elaborate the process for creating a Modelica model for the studied system; Last, we discuss the two proof-of-concept cases and future work.

\section{Studied System}

Figure 1 is a schematic of the studied system. The studied system is defined to represent how a typical HVAC system interacts with the power grid, and consists of three subsystems:

1) Electrical Subsystem. This subsystem represents the power system and provides power to the rest of the studied system. It has one variable frequency drive (VFD), which adjusts the frequency of power based on the request from the building system.

2) Mechanical Subsystem. This subsystem represents the process of converting to the service that building systems provide to occupants. In our case, the service we considered is cooling/heating demand. The mechanical system contains two components: an induction motor that creates torque given receiving power flow and a transitional device that converts the torque to the mechanical work.

3) Thermal Subsystem. This subsystem represents the HVAC system that addresses the cooling/heating demand in buildings. It has motor-driven devices such as a fan/pump that delivers fluid flows such as air or water flow to actually remove/add heat from/to the indoor environment. There are also feedback-loop controls which guarantee that the motor-driven device delivers desired flow rates by adjusting the frequency of the VFD.

It is noted that both the electrical subsystem and the thermal subsystem are treated as abstract interfaces to be connected to more detailed models for power systems or building systems. Details of the power system or the building systems such as the power distribution flow are beyond the scope of this study.

\section{System Model}

As discussed in the previous section, the electrical subsystem and the thermal subsystem are treated as interfaces to be connected to the power system and buildings, respectively. Thus, when elaborating on the system model, we mainly focus on the mechanical subsystem. For the details regarding the modeling of the power system or buildings, readers can find more information in (Chassin et al., 2008; Crawley et al., 2001).

In the mechanical subsystem, one major equipment is the induction motor. Although Modelica Standard Library has existing models for the induction machine, they are built on an electrical interface that are hardly compati- ble with Modelica Buildings library (Wetter et al., 2014) that is widely used to perform dynamic simulation on the building side. Whatmore, those induction machine model are too complicated for this preliminary study. Therefore, in this paper, we presented a new induction motor model which can be sufficient to capture the dynamics we need for Building-to-Grid integration, and utilized the electrical interface in Modelica Buildings Library to easily couple with building side.

In the presented induction motor model, the inputs include voltage, $V$, frequency, $f$, and load torque, $\tau_{L}$, while the output is the electromagnetic torque, $\tau_{e}$. The major parameters include the number of the pole pair, $n_{p}$, the number of the phase, $n$, the moment of the inertia, $J_{m}$, the electric resistance of the stator, $R_{s}$, the electric resistance of the rotor, $R_{r}$, the complex component of the impedance of the stator, the complex component of the impedance of rotor, and the complex component of the magnetizing reactance, $X_{s}, X_{r}$, and $X_{m}$.

$\tau_{e}$ is calculated by solving the following equations

$$
\begin{array}{r}
\tau_{e}=\frac{n\left(V \frac{X_{m}}{X_{m}+X_{s}}\right)^{2} \frac{R_{r}}{s}}{\omega_{s}\left(\left(R_{s}\left(\frac{X_{m}}{X_{m}+X_{s}}\right)^{2}+\left(\frac{R_{r}}{s}\right)^{2}\right)\right)^{2}+\left(X_{r}+X_{s}\right)^{2}} \\
\omega_{s}=\frac{4 \pi f}{n_{p}} \\
s=\frac{\omega_{s}-\omega_{r}}{\omega_{s}} \\
\frac{d \omega_{r}}{d t}=\frac{\tau_{e}-\tau_{L}}{J_{m}}
\end{array}
$$

In addition, the active and the reactive power of the motor, $P$ and $Q$, are calculated by

$$
\begin{array}{r}
P=\frac{n V^{2} R_{e q}}{R_{e q}{ }^{2}+X_{e q}{ }^{2}} \\
Q=\frac{n V^{2} X_{e q}}{R_{e q}{ }^{2}+X_{e q}{ }^{2}} \\
R_{e q}=R_{s}+\frac{R_{r} s X_{m}^{2}}{R_{r}^{2}+\left(s^{2}\right)\left(X_{r}+X_{m}\right)^{2}} \\
X_{e q}=X_{s}+\frac{X_{m}\left(R_{r}^{2}+\left(s X_{r}\right)^{2}+\left(s^{2}\right) X_{r} X_{m}\right)}{R_{r}^{2}+\left(s^{2}\right)\left(X_{r}+X_{m}\right)^{2}}
\end{array}
$$

Regarding the transitional device, its inputs include $\tau_{e}$ and the load shaft power $P_{\text {shaft }}$ while the outputs include the load speed, $\omega_{r}$ and $\tau_{L}$. One major parameter for the transitional device is the load moment inertia $J_{L} . \tau_{L}$ is calculated by solving the following equations:

$$
\begin{array}{r}
\tau_{L}=\frac{P_{\text {shaft }}}{\omega_{r}} \\
\frac{d \omega_{r}}{d t}=\frac{\tau_{e}-\tau_{L}}{J_{L}}
\end{array}
$$




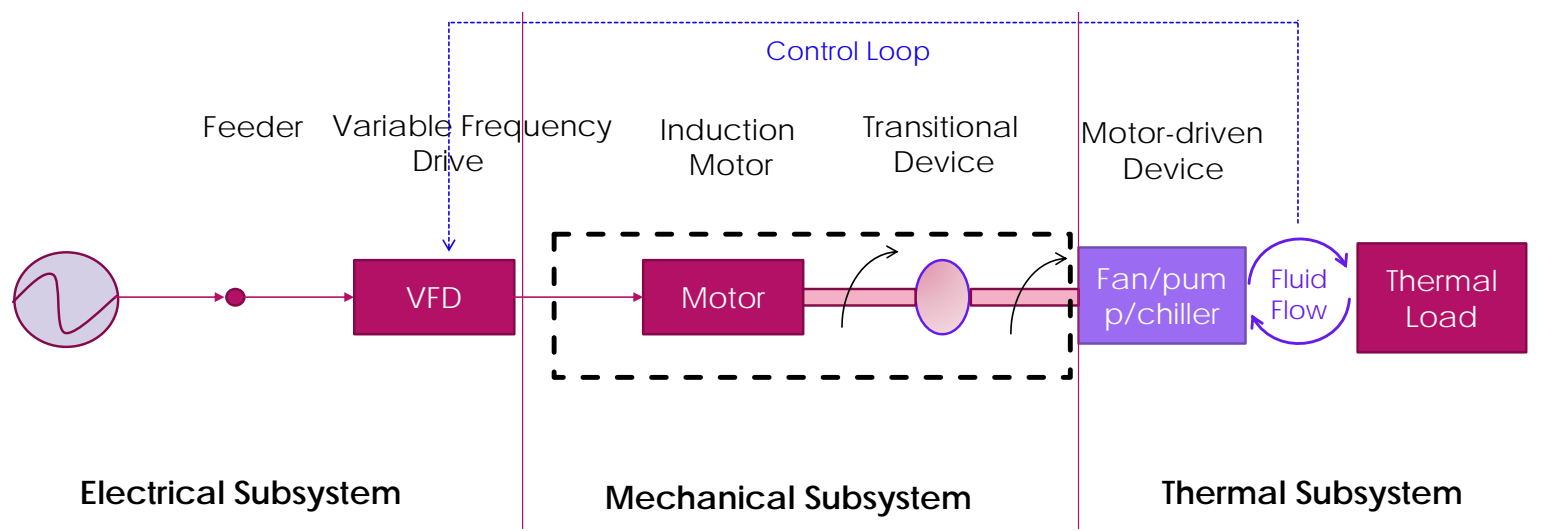

Figure 1. The studied system

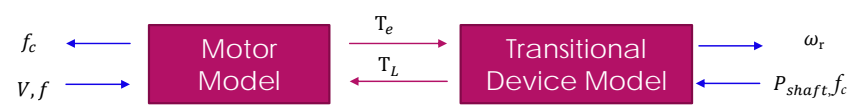

Figure 2. The input-output interface for the generated Modelica model

The load shaft power $P_{\text {shaft }}$ can be calculated from a conventional pump model commonly used in building simulation tools.

$$
P_{\text {shaft }}=\frac{\Delta p Q}{\eta_{\text {shaft }}}
$$

$\Delta p$ and $\eta_{\text {shaft }}$ are the head and shaft efficiency of the pump. They can be expressed as a quadratic equation in terms of volume flowrate $Q$ and normalized rotation speed $r$.

$$
\begin{aligned}
\Delta p & =\left(a_{0}+a_{1}\left(\frac{Q}{r}\right)+a_{2}\left(\frac{Q}{r}\right)^{2}\right) r^{2} \\
\eta_{\text {shaft }} & =\left(b_{0}+b_{1}\left(\frac{Q}{r}\right)+b_{2}\left(\frac{Q}{r}\right)^{2}\right) r^{2}
\end{aligned}
$$

where the normalized speed $r$ can be calculated by receiving rotation speed $\omega_{r}$ from the transitional device, and the known nominal rotation speed $\omega_{r, 0}$.

$$
r=\frac{\omega_{r}}{\omega_{r, 0}}
$$

We then implemented the above-mentioned models of the induction motor and the transitional device in Modelica (Fritzson and Engelson, 1998). Modelica is an equation-based modeling language that allows the systems to be described with implicit equations. Therefore, equations (3) to (12) can be used directly to create the corresponding Modelica codes. The generated Modelica models have the input-output interface shown as Figure 2. The inputs include the load shaft power and the frequency control signal, $f_{c}$, from buildings, as well as the voltage $V$ and the actual frequency $f$ from the power system; the outputs

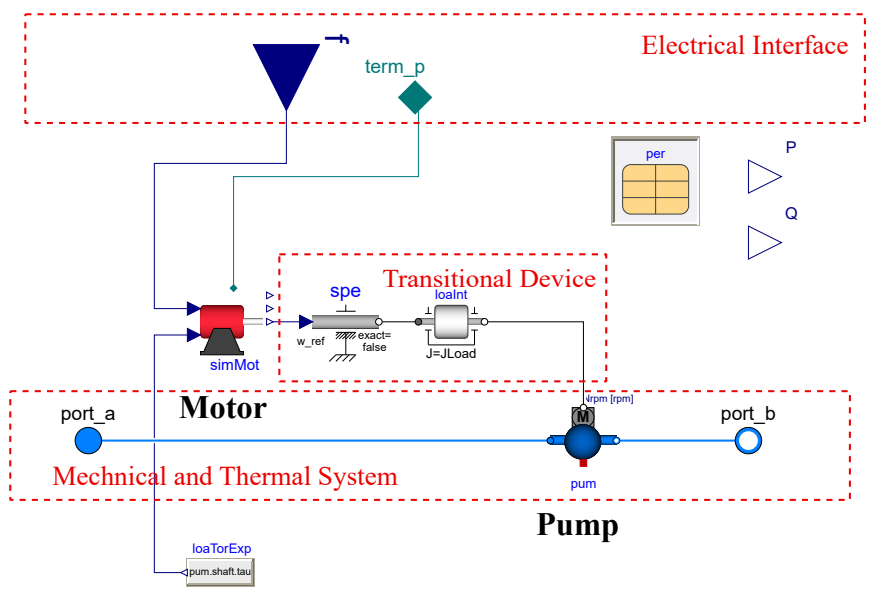

Figure 3. Modelica representation of the motor-driven pump model

are the actual speed of the motor-driven devices $\omega_{r}$ and the actual frequency control signal to the power system.

The final motor-driven pump model is built by connecting the motor model, transitional device, and a mechanical pump model in Modelica Buildings library together. The Modelica representation is shown in Figure 3. The electrical interface is used to connect with the grid model to receive electrical information. The received electrical information is then delivered to the induction motor model, which generates electromagnetic torque that is transmitted to the mechanical pump by the transitional device.

\section{Proof-of-concept Study}

We conducted one case study to demonstrate how the generated models can capture the interactions between the power system and the buildings. In this case, we considered a simplified hydraulic cooling system. As shown in 4, a pump on the supply side delivers cold water between an ideal cooling source and an air handler. The cooling source maintains the temperature of the leaving water to be $7{ }^{\circ} \mathrm{C}$ while the water flow rate is modulated to maintain the air leaving the air handler to be around $16^{\circ} \mathrm{C}$. The change in water flow rate is realized by adjusting the valve 


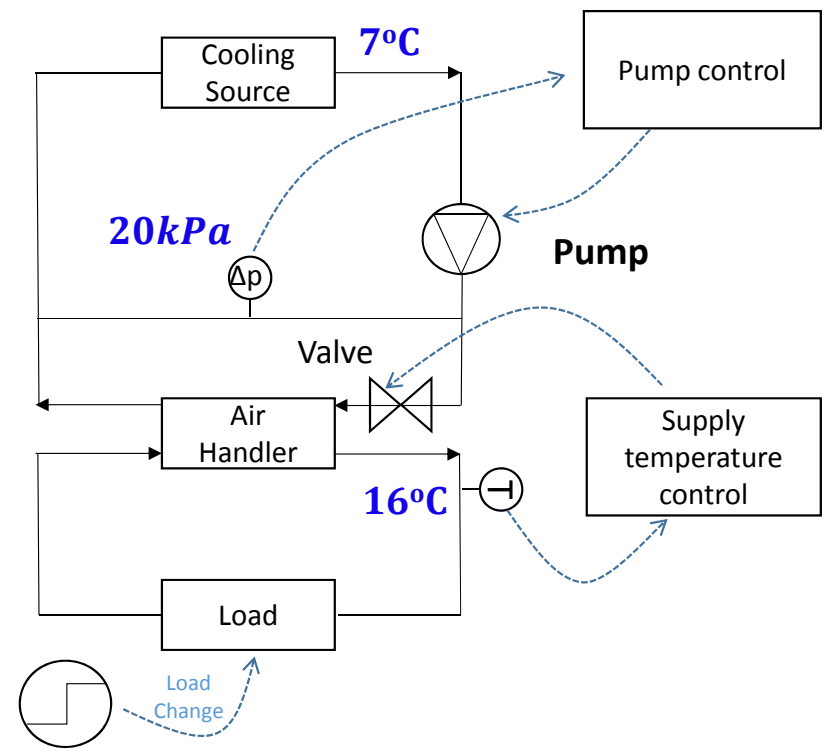

Figure 4. The schematic drawing of the proof-of-concept case

portion of a two-way valve. The frequency of the pump is adjusted to maintain a constant pressure difference in the pipe across the air handler.

We implemented the above case based on the Modelica Buildings library, which has been demonstrated to be able to perform building level energy modeling and simulation (Huang et al., 2016, 2017; Fu et al., 2018, 2019). For example, the Buildings.Fluid.Sources.BoundarypT is used to model the ideal cooling source. In addition, to model the pump, we consider three modules: One (named "conventional pump") is the pump module from the Modelica Buildings Library (Buildings.Fluid.Movers.BaseClasses.PartialFlowMachine).

This model doesn't take voltage into consideration. The second one (named "proposed pump") is modified based on the first one by adding the developed induction motor and transitional device. Figure 5 shows a diagram of the generated Modelica model with the proposed pump in Dymola environment (Brück et al., 2002). The performance characteristics curve of the pump is from the Modelica Buildings Library.

We simulated the Modelica model for two different scenarios. In the first scenario, we studied how the system behaves when we change the status of the pump from off to on. In this case, as shown in Figure 6, for both the "conventional pump" and the "proposed pump", the pressure difference starts with $0 \mathrm{kPa}$ and approaches the set point of $20 \mathrm{kPa}$. However, it takes around 60s for the "proposed pump" to be quite close to the set point while more than 400s for the "conventional pump" to reach to the similar value. On the same time, we also observe much higher pump power from 0 to 60 s in the simulation of the "proposed pump". But as soon as the pressure is close to the set point, the simulated power from the "proposed pump" quickly drops to a value that is closer to that from the "con-

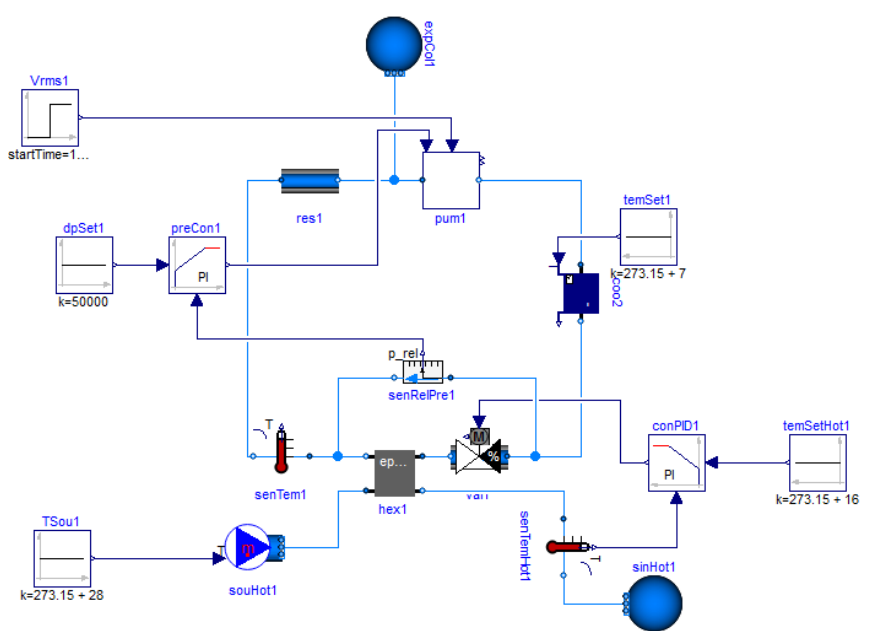

Figure 5. The diagram of the Modelica model in the proof-ofconcept case

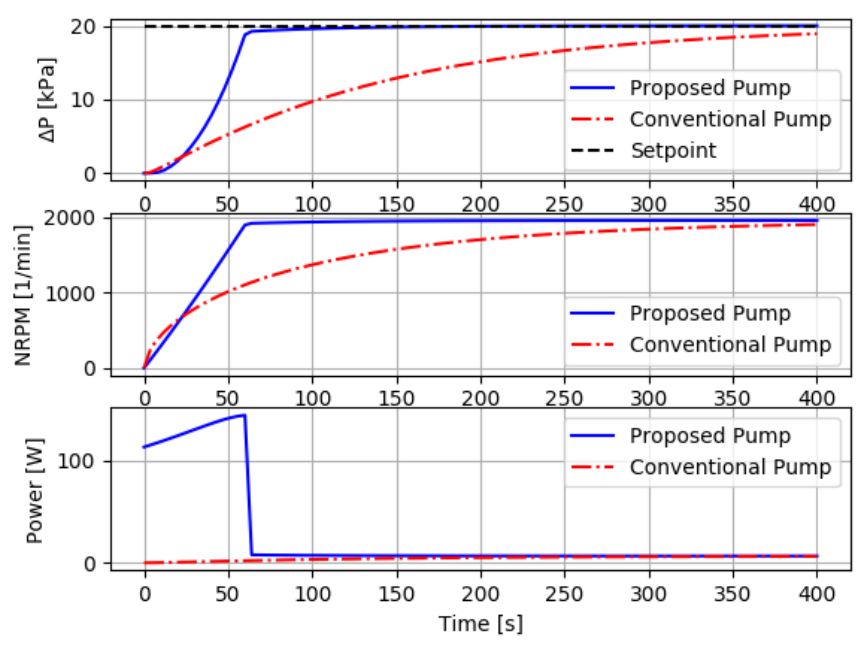

Figure 6. Simulation results for the first scenario

ventional pump". It is our view that the simulated result from the "proposed pump" is closer to what we observed in the real world, in terms of the power change.

In the second scenario, we studied how the system behaves when we change the supply voltage. To better evaluate the performance of the "proposed pump", we considered another option for modelling the pump as a reference. In this option, the induction motor and transitional device in the "proposed pump" are replaced with the ZIP coefficient model as described in equation 1 and 2 . The resulting new model is named "ZIP pump". Figure 7 shows the simulation results for the second scenario. The supply voltage is kept constant before 100s. In that case, all three models generate identical results in terms of active power. At $t=100 \mathrm{~s}$, the voltage changes from $120 \mathrm{~V}$ to around $108 \mathrm{~V}$; the active pump power in the "conventional pump" is unchanged. For the "ZIP pump", the active pump power immediately changes to a smaller value while that in "proposed pump" decreases at first and then takes around 11s to increase to the same value as predicted in the "ZIP 

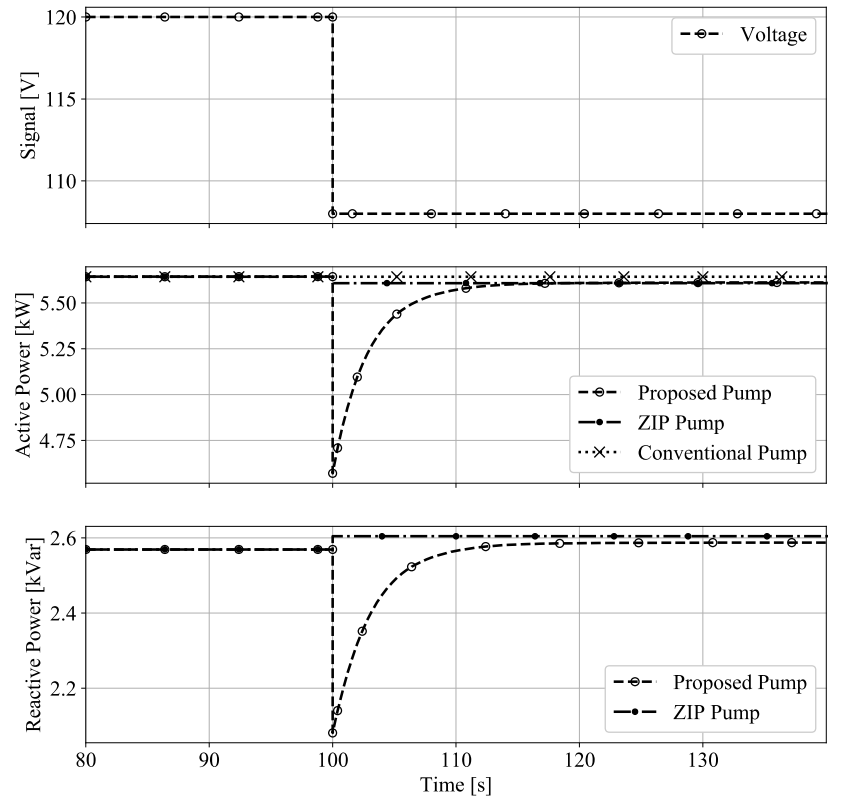

Figure 7. Simulation results for the second scenario

pump". Regarding the reactive power simulation result, we see a pattern similar to that of the active power. Based on the results, it is clear that the "proposed pump" provides a more realistic representation of the response of the building systems to the changing supply voltage.

\section{Conclusion}

In this paper, we developed Modelica models enable consideration the interactions between buildings and the power system. Based on the result from the proof-ofconcept study, we can see that the proposed models can provide much better representation of the response of the building systems to changing operation status and changing supply voltage. Therefore, we believe those models can support research where more complicated interaction between buildings and the power system need to be considered.

In a future study, we will perform the validation of the proposed models with real-world data to quantitatively evaluate their performance. In addition, the motor model will also be connected with power flow analysis tools instead of receiving ideal grid information as in the case study. This will enable a simultaneous simulation of building and grid system.

\section{Acknowledgement}

This work has been supported by the the Buildings Technologies Office of the U.S. Department of Energy's Office of Energy Efficiency and Renewable Energy.

The authors would like to thank Dr. Marina Sofos, Sensors and Controls Technology Manager in the Building Technologies Office at the the U.S. Department of Energy, for her technical guidance and support.

The authors thank Dr. Thomas McDermott for his help- ful technical feedback and Matthew Wilburn for editing the final version of the manuscript.

\section{References}

Aainaa Mohd Arriffin, Muhammad Murtadha Othman, Amirul Asyraf Mohd Kamaruzaman, Ismail Musirin, Ainor Yahya, and Mohd Fuad Abdul Latip. Stochastic approach of voltage optimization to maximize power saving in a building. Indonesian Journal of Electrical Engineering and Computer Science, 8(1):268-272, 2017.

Andrii Bichik et al. Impact of voltage variation on domestic and commercial loads. 2015.

A. Bokhari, A. Alkan, R. Dogan, M. Diaz-Aguilâş, F. de LeÃşn, D. Czarkowski, Z. Zabar, L. Birenbaum, A. Noel, and R. E. Uosef. Experimental determination of the zip coefficients for modern residential, commercial, and industrial loads. IEEE Transactions on Power Delivery, 29(3):1372-1381, June 2014.

Dag Brück, Hilding Elmqvist, Sven Erik Mattsson, and Hans Olsson. Dymola for multi-engineering modeling and simulation. In Proceedings of modelica, volume 2002. Citeseer, 2002.

David P Chassin, K Schneider, and C Gerkensmeyer. Gridlab-d: An open-source power systems modeling and simulation environment. In Transmission and distribution conference and exposition, 2008. $t \& d$. IEEE/PES, pages 1-5. IEEE, 2008.

Drury B Crawley, Linda K Lawrie, Frederick C Winkelmann, Walter F Buhl, Y Joe Huang, Curtis O Pedersen, Richard K Strand, Richard J Liesen, Daniel E Fisher, Michael J Witte, et al. Energyplus: creating a new-generation building energy simulation program. Energy and buildings, 33(4):319-331, 2001.

Peter Fritzson and Vadim Engelson. Modelica - a unified objectoriented language for system modeling and simulation. In European Conference on Object-Oriented Programming, pages 67-90. Springer, 1998.

Yangyang Fu, Michael Wetter, and Wangda Zuo. Modelica models for data center cooling systems. In 2018 Building Performance Analysis Conference and SimBuild, Chicago, Illinois, United States of America, 2018.

Yangyang Fu, Wangda Zuo, Michael Wetter, James VanGilder, $\mathrm{Xu}$ Han, and David Plamondon. Equation-based objectoriented modeling and simulation for data center cooling: A case study. accepted by Energy and Buildings, 2019.

Elliott M Gilbert. Regulated power supply, July 27 1965. US Patent 3,197,691.

K. Hatipoglu, I. Fidan, and G. Radman. Investigating effect of voltage changes on static zip load model in a microgrid environment. In 2012 North American Power Symposium (NAPS), pages 1-5, Sept 2012.

X. He, R. Zhao, C. Zhu, and H. Yang. Improving short-term voltage stability problems by variable-speed air-conditioners. In 2012 15th International Conference on Electrical Machines and Systems (ICEMS), pages 1-6, Oct 2012. 
GK Hood. The effects of voltage variation on the power consumption and running cost of domestic appliances. In Australasian Universities Power Engineering Conference ( $A U$ PEC), 2004.

Sen Huang, Wangda Zuo, and Michael D Sohn. Amelioration of the cooling load based chiller sequencing control. Applied Energy, 168:204-215, 2016.

Sen Huang, Wangda Zuo, and Michael D Sohn. Improved cooling tower control of legacy chiller plants by optimizing the condenser water set point. Building and Environment, 111: 33-46, 2017.

Keun H Lee. Optimization of a hybrid electric power system design for large commercial buildings: an application design guide. PhD thesis, Colorado School of Mines. Arthur Lakes Library, 2014.

Dezhi Li, Gaoying Cui, Lingling Sun, Jiru Yang, Ciwei Gao, and Xiao Chen. A control strategy for static voltage stability based on air conditioner load regulation. In Systems and Informatics (ICSAI), 2017 4th International Conference on, pages 288-293. IEEE, 2017.

Michael Wetter, Wangda Zuo, Thierry S Nouidui, and Xiufeng Pang. Modelica buildings library. Journal of Building Performance Simulation, 7(4):253-270, 2014.

Bei Wu, Yan Zhang, and Minjiang Chen. The effects of air conditioner load on voltage stability of urban power system. In 6 th WSEAS International Conference on Power Systems(PE'06), volume 6. Citeseer, 2006. 